\title{
Programas de entrenamiento neuromuscular para la prevención de lesiones en jóvenes deportistas. Revisión de la literatura
}

\author{
Neuromuscular training programs for the injury \\ prevention in young athletes. A literature review
}

\author{
Francisco Javier Robles-Palazón y Pilar Sainz de Baranda \\ Facultad de Ciencias del Deporte. Universidad de Murcia. Campus de Excelencia Internacional Regional "Campus Mare Nostrum".
}

\begin{abstract}
Resumen: Los objetivos de esta revisión son: (1) describir los principales programas de prevención de lesiones de la extremidad inferior en jóvenes deportistas que cuentan con una eficacia demostrada, cuya prevención ha estado dirigida a deportes colectivos de balón, no requieren de material adicional para su aplicación y la duración permite su implementación como calentamiento previo a la práctica deportiva; y (2) analizar las ventajas e inconvenientes de las medidas preventivas de acuerdo a su posibilidad de implantación de manera regular en el deporte. Para ello, un total de 5 programas neuromusculares para la prevención de lesiones fueron seleccionados. Aunque todos los programas han demostrado efectos positivos sobre la reducción de la incidencia lesional, el análisis pormenorizado de las características de las 5 medidas preventivas recomienda el uso de los programas FIFA 11+ y el Knäkontroll por su mayor adaptabilidad al contexto de aplicación y la facilidad de su implementación de manera regular en el deporte. Palabras clave: calentamiento, lesiones, FIFA 11+, adolescentes, fútbol.
\end{abstract}

Abstract: The aims of this review are: (1) describing the principal lower extremity injury prevention programs in young athletes that have a demonstrated efficacy, whose prevention have been destined for ball team sports, do not require any additional material for their application and the duration allows for their implementation as previous warm-up programs; and (2) analyzing the advantages and disadvantages of the prevention strategies according to their possibility of implantation in the regular sport practice. For this purpose, a total of 5 neuromuscular injury prevention programs were selected. Although all the programs have shown positive effects in terms of the reduction of sport injury incidence, the detailed analysis of the characteristics of the prevention strategies recommend the utilization of the FIFA 11+ and Knäkontroll programs for their greater adaptability to the application context and their facility for the implementation in the regular sport practice.

Keywords: warm-up, injuries, FIFA 11+, adolescents, soccer.

\section{Introducción}

La práctica deportiva de manera regular ha sido considerada un componente de vital importancia para mantener un estilo de vida activo y saludable, que reduzca el riesgo de sufrir numerosas enfermedades (tales como la obesidad, diabetes, hipertensión, etc. [Janssen \& Leblanc, 2010; Loprinzi, Cardinal, Loprinzi, \& Lee, 2012]) y ayude a mantener un estado de bienestar físico, psíquico y social en niños y adolescentes. Sin embargo, el incremento de las demandas físicas del deporte, unido a los desajustes en el control motor propios del periodo puberal, colocan a los participantes de actividades deportivas en una situación más vulnerable para sufrir una lesión en comparación con sus homólogos no deportistas (Maffulli, Longo, Gougoulias, Loppini, \& Denaro, 2010). En este sentido, las lesiones podrían contrarrestar los efectos positivos que la participación deportiva genera en el niño y/o adolescente si se ve forzado a abandonar la misma como consecuencia de los efectos residuales derivados de las lesiones (Longo, Lamberti, Maffulli, \& Denaro, 2011).

Dada la gran magnitud de este problema, parece justifica-

Dirección para correspondencia [Correspodence address]: Francisco Javier Robles-Palazón. franciscojavier.robles1@gmail.com da la necesidad de desarrollar medidas preventivas destinadas a reducir el número y el impacto que las lesiones poseen en el mundo del deporte. Así, en los últimos años, ha proliferado exponencialmente la publicación de distintos programas que tienen por objetivo la disminución de esta incidencia lesional, especialmente en extremidades inferiores (lesiones más frecuentes [Hootman, Dick, \& Agel, 2007]). Prestigiosos centros, asociaciones y universidades, como el Centro Médico y de Investigación de la FIFA, el Centro Deportivo Traumatológico y de Investigación de Oslo o la Fundación de Medicina Deportiva de Santa Mónica, han empleado sus principales recursos en investigar y divulgar programas preventivos que sean efectivos y que, además, puedan ser implementados por todos los profesionales del deporte sin necesidad de grandes recursos humanos, materiales y temporales. No obstante, y a pesar de que muchas de las medidas propuestas han demostrado reducir significativamente el número de lesiones deportivas, la tasa de incidencia lesional en muchos deportes parece no haber disminuido en lo largo de los últimos ańos (Beachy \& Rauh, 2014; Hootman et al., 2007).

Quizás, una de las razones que explique esta discordancia entre el incremento de la publicación de medidas preventivas y el mantenimiento de ratios de incidencia lesional 
estables podría ser el habitual desconocimiento o desfase temporal existente entre los avances y evidencias científicas obtenidas como resultado de las numerosas investigaciones realizadas y los métodos de entrenamiento aplicados en la práctica profesional. Por ello, es de vital importancia incrementar la divulgación de las distintas propuestas desarrolladas para que los entrenadores y preparadores físicos conozcan su contenido, su desarrollo y, por supuesto, su eficacia. Además, el incremento del volumen de información publicada en las últimas décadas también puede dificultar el análisis y selección por los profesionales de aquellas estrategias más adecuadas para su implementación en el día a día del deporte. Por todo ello, los objetivos de esta revisión bibliográfica son: (1) describir los principales programas de prevención de lesiones de la extremidad inferior en jóvenes (<19 años) deportistas que cuentan con una eficacia demostrada, y cuya prevención ha estado dirigida a deportes colectivos de balón, no requiere de material adicional para su aplicación, y la duración permite su implementación como calentamiento previo a la práctica deportiva; y (2) analizar las ventajas e inconvenientes de las medidas preventivas de acuerdo a su posibilidad de implantación de manera regular en el deporte.

\section{Descripción de los programas de prevención de lesiones}

\section{FIFA $11+$}

El propósito del programa FIFA 11+, diseñado por el Centro Médico y de Investigación de la FIFA (F-MARC) junto con el Centro Deportivo Traumatológico y de Investigación de Oslo, y publicado por Soligard et al. (2008), es la prevención de lesiones de la extremidad inferior en jóvenes jugadores de fútbol. El FIFA 11+ es un programa multicomponente, que incluye varios ejercicios dinámicos y que están focalizados en los principales factores de riesgo intrínsecos y modificables de las lesiones que acontecen en las extremidades inferiores. El protocolo se divide en tres apartados: (1) ejercicios de carrera a baja intensidad, (2) fuerza, pliometría y equilibrio, y (3) ejercicios de carrera combinados con movimientos específicos del fútbol, que suman un total de 15 ejercicios y cuya duración aproximada de su completa implementación es de 20 minutos (Tabla 1).

De los programas incluidos en esta revisión, el FIFA 11+ es, seguramente, el que ha contado con mayor difusión y popularidad entre los distintos profesionales del deporte, debido no solo al reconocimiento y prestigio que presentan los organismos encargados de su desarrollo y publicación, sino también a la gran eficacia demostrada sobre la reducción del número de lesiones en distintas poblaciones y contextos. Así, la aplicación de este programa preventivo con una frecuencia mínima de dos veces a la semana durante al menos 3 meses consecutivos ha mostrado efectos positivos sobre la tasa de incidencia lesional en mujeres (Soligard et al., 2008; Steffen et al., 2013) y hombres (Owoeye, Akinbo, Tella, \& Olawale, 2014) jóvenes futbolistas. Además, la aplicación sistemática de este programa podría ayudar, incluso, a reducir la incidencia lesional en otras cohortes poblacionales (e.g. jugadores de baloncesto [Longo et al., 2012]), aunque la población objetivo de sus autores hayan sido los futbolistas.

Con el objetivo de incrementar los beneficios obtenidos de la implementación con el FIFA 11+, se recomienda mantener una elevada adherencia al programa durante los periodos de intervención, desarrollando todos los ejercicios del mismo en todas las sesiones de entrenamiento en las que se aplique. En este sentido, los datos aportados por Steffen et al. (2013) mostraron mejoras en la tasa de reducción de lesiones entre aquellos deportistas que presentaron una alta adherencia al programa (definida como el número de ejercicios total del FIFA 11+ realizados por cada jugador a lo largo de la temporada) frente a aquellos que presentaron media o baja adherencia. 
Tabla 1. Descripción de ejercicios y duración del programa FIFA 11+.

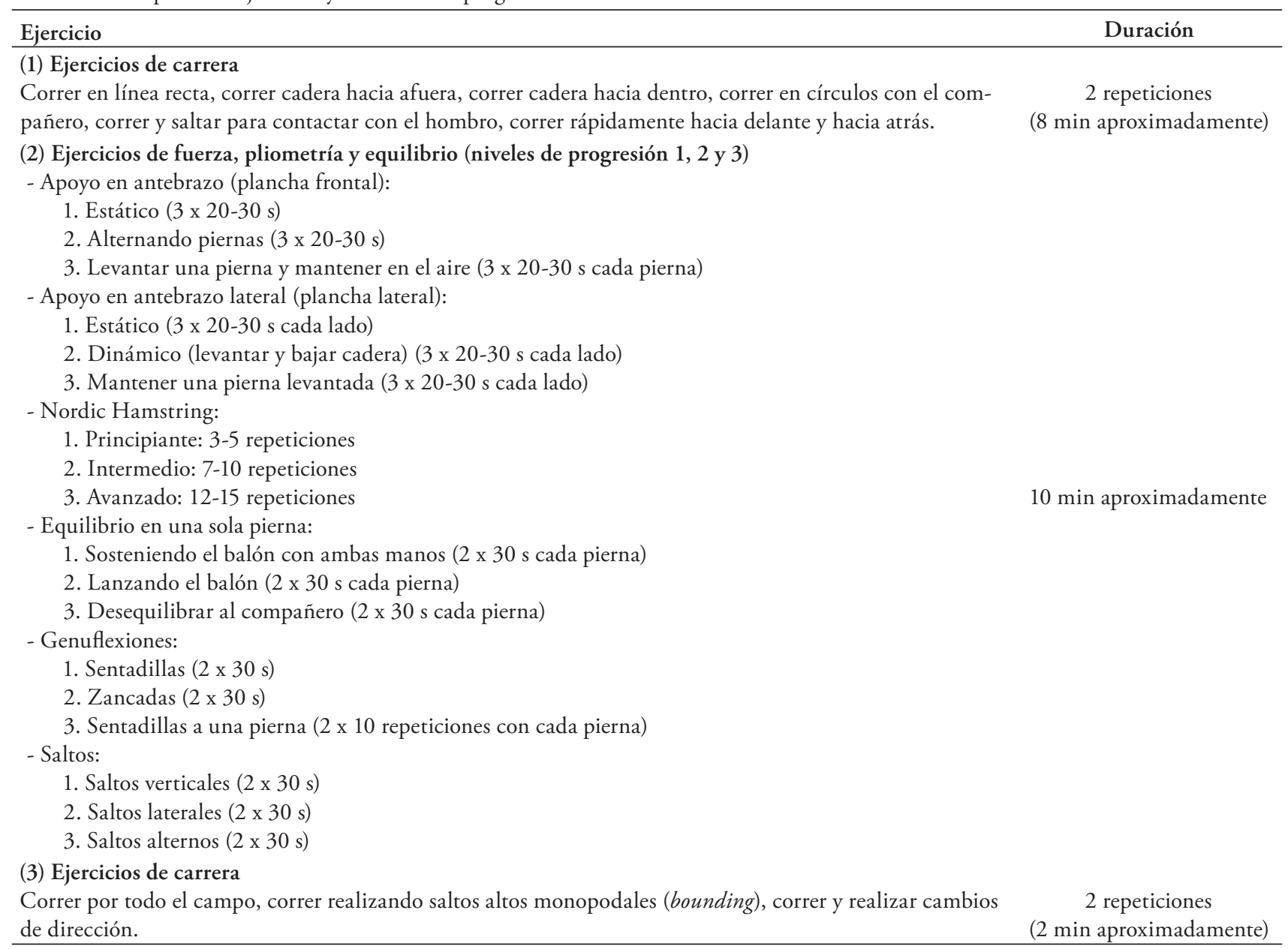

\section{Harmoknee}

El programa de prevención de lesiones publicado por Kiani et al. (2010) tiene por objetivo la reducción de la ratio de incidencia lesional en la articulación de la rodilla en jóvenes jugadores de fútbol. Para ello, el programa Harmoknee focaliza la atención en la mejora de los principales factores de riesgo modificables de las lesiones de rodilla, y más específicamente en aquellos relacionados con los desgarros y rupturas del LCA (e.g. estabilidad dinámica global de la extremidad inferior, control de la rodilla durante acciones de salto y caída, fuerza excéntrica, rango de movimiento articular,...). Se trata de un programa multicomponente, compuesto por 5 apartados o bloques de ejercicios bien definidos y seleccionados de acuerdo a la evidencia científica existente, así como a la extensiva experiencia práctica de numerosos entrenadores y profesionales del mundo del fútbol (Tabla 2).

La popularidad de este programa de calentamiento en el mundo del fútbol ha experimentado un enorme crecimiento en los últimos años gracias a su sencillez, austeridad de los recursos materiales (no precisa de material adicional) y temporales (duración total de su implementación de 20-25 minutos) que se precisan para su puesta en marcha y, sobre todo, a la eficacia demostrada en la reducción del número de lesiones de rodilla registradas durante un año deportivo. No obstante, y aunque los efectos que se derivan de su implementación sobre determinados parámetros del rendimiento motor han sido analizados en varias publicaciones previas (Daneshjoo, Mokhtar, Rahnama, \& Yusof, 2012, 2013; Daneshjoo, Rahnama, Mokhtar, \& Yusof, 2013), la eficacia en términos de disminución de la incidencia lesional solo ha sido comprobada en mujeres jugadoras de fútbol de 13-19 años y por un único estudio (Kiani et al., 2010). Por tanto, se hace necesario conocer los efectos derivados de la intervención con este programa en otras poblaciones así como corroborar los datos aportados por estos autores en futuras investigaciones. 
Tabla 2. Descripción de ejercicios y duración del programa HarmoKnee.

\begin{tabular}{lc}
\hline Ejercicio & Duración \\
\hline (1) Calentamiento & \\
Carrera ( $\geq 4-6$ min), carrera hacia atrás apoyando tercio distal del pie (aprox. 1 min), skipping (aprox. 30 s), técnica de presión & \\
defensiva (aprox. 30 s), uno y uno: carrera zigzag hacia delante y técnica de presión defensiva en zigzag hacia atrás ( $\geq 2$ min). & $\geq 10$ min \\
(2) Activación muscular & Aproximada- \\
Activación de gemelos, cuádriceps, isquiosurales, flexores de cadera, aductores, lumbares y musculatura de la cadera & mente 2 min \\
(aprox. 4 s para cada pierna/lado). & Aproximada- \\
(3) Equilibrio & mente 2 min \\
Salto horizontal bipodal antero-posterior, salto lateral unipodal, salto horizontal unipodal antero-posterior, salto vertical & Aproximada- \\
bipodal con o sin balón (aprox. 30 s por ejercicio). & mente 4 min \\
(4) Fuerza & Aproximada- \\
Zancadas en el sitio, curl de isquiosural en parejas, sentadillas unipodales (aprox. 1 min por ejercicio). & mente 3 min \\
(5) Estabilidad del core &
\end{tabular}

\section{KIPP}

El programa de prevención de lesiones de la rodilla (Knee Injury Prevention Program ${ }^{\circledR}$ [KIPP]) publicado por LaBella et al. (2011) tiene por objetivo disminuir el número de lesiones de LCA entre los jóvenes deportistas. Para ello, el programa cuenta con una amplia variedad de ejercicios que combinan el trabajo de contenidos como la movilidad dinámica, la fuerza, la pliometría y la agilidad. Con el objetivo de favorecer la progresión y variabilidad de la medida propuesta, los autores presentan, en su propuesta preventiva publicada en el ańo 2011 y en la que centraremos el análisis por ser la que ha demostrado su eficacia, una distribución de cargas y ejercicios distintos en función de la semana de aplicación del programa (Tabla 3). Sin embargo, según la información aportada por los propios autores, actualmente el programa que se está desarrollando bajo el nombre de KIPP cuenta con algunas modificaciones en la distribución y tipo de ejercicios (Tabla 4).

Los efectos derivados de la aplicación sistemática de este programa como calentamiento previo a la práctica deportiva sobre la incidencia lesional parecen ser positivos. Los resultados obtenidos por LaBella et al. (2011) mostraron una reducción significativa de las lesiones producidas en la extremidad inferior en el grupo KIPP en comparación con el grupo control. Los datos en cuanto a la reducción de la lesión de LCA (lesión objetivo del programa) también fueron positivos para el grupo de intervención; mientras que la ratio de lesión de LCA del grupo KIPP fue de 0.07 lesiones/1000h de exposición a la práctica, el grupo control obtuvo 0.26 lesiones $/ 1000$ h de exposición. Sin embargo, este descenso no fue estadísticamente significativo $(p=.09)$.

Tabla 3. Descripción de ejercicios y duración del programa KIPP.

\begin{tabular}{|c|c|}
\hline Ejercicios comunes para todas las semanas & $\begin{array}{l}\text { Repeticiones / } \\
\text { Duración }\end{array}$ \\
\hline $\begin{array}{l}\text { (1) Carrera } \\
\text { Dos vueltas a la pista o una vuelta al campo }\end{array}$ & \\
\hline $\begin{array}{l}\text { (2) Movilidad dinámica } \\
\text { A) Desplazamientos: } \\
\text { Carrera, skipping, carioca, desplazamiento lateral, sprint } 75 \% \text { intensidad, skipping rodillas altas, carioca rodillas } \\
\text { altas, sprint } 100 \% \text { intensidad, carrera hacia atrás, spiderman (bear crawl), talón-glúteo, carrera hacia atrás con } \\
\text { giro y sprint, skipping diagonal }\end{array}$ & 2.A) $100 \mathrm{ft} /$ ejercicio \\
\hline $\begin{array}{l}\text { B) Circunducción de brazos (anterior y posterior) } \\
\text { C) Rotación de tronco } \\
\text { D) Elevaciones de piernas (swings) } \\
\text { Frontal, lateral }\end{array}$ & $\begin{array}{l}\text { 2.B) } 20 \mathrm{reps} / \mathrm{brazo} \\
\text { 2.C) } 10 \mathrm{reps} / \mathrm{lado} \\
\text { 2.D) } 10 \mathrm{reps} / \text { pierna } \\
\text { por ejercicio }\end{array}$ \\
\hline $\begin{array}{l}\text { (5) Agilidad } \\
\text { A) Carrera aceleración-deceleración (shuttle run) } \\
\text { B) Carrera diagonal (zig-zag) } \\
\text { C) Desplazamientos laterales }\end{array}$ & $\begin{array}{l}\text { 5.A) } 50 \mathrm{ft} \times 10 \mathrm{reps} \\
\text { 5.B) } 50 \mathrm{ft} \times 10 \mathrm{reps} \\
\text { 5.C) } 15 \mathrm{ft} \times 10 \mathrm{reps}\end{array}$ \\
\hline
\end{tabular}




\section{Ejercicios semana 1}

Duración

\section{(3) Fortalecimiento}

Elevación de talones, sentadillas, plancha frontal y lateral, flexiones, zancadas frontales, superman para lumbares, superman alternando elevación brazo-pierna contralateral.

(4) Pliometría

Multi-saltos (flexo-extensión de tobillo) ${ }^{1}$, saltos con rodillas al pecho ${ }^{1}$, saltos con giro $180^{\circ 1}$, squat jumps ${ }^{1}$, salto horizontal bipodal con caída controlada (5 reps), saltar 3 conos (frontal y lateral) ${ }^{1}$, saltos unipodales profundos (bounding) en el sitio'.

\begin{tabular}{l} 
Ejercicios semana 2 \\
\hline (3) Fortalecimiento \\
Elevación de talones, sentadillas, plancha frontal y lateral, flexiones, zancadas laterales y diagonales, superman \\
para lumbares, superman alternando elevación brazo-pierna contralateral, superman levantando piernas $\left(90^{\circ}\right.$ \\
rodillas) y brazos. \\
(4) Pliometría \\
Multi-saltos (flexo-extensión de tobillo) ${ }^{1}$, saltos con rodillas al pecho ${ }^{1}$, squat jumps ${ }^{1}$, saltar 3 conos (frontal y \\
lateral $)^{1}$, zancadas alternas con salto (salto de tijera $)^{1}$, saltos laterales profundos (bounding $)^{1}$, doble salto unipodal \\
y caída controlada $(5$ reps/pierna), tres multi-saltos y salto vertical profundo $(5$ reps).
\end{tabular}

\section{Ejercicios semana 3}

\section{(3) Fortalecimiento}

Elevación de talones, sentadillas, plancha frontal y lateral, flexiones, zancadas laterales y diagonales, zancadas con desplazamiento, superman para lumbares, superman alternando elevación brazo-pierna contralateral, superman levantando piernas ( $90^{\circ}$ rodillas) y brazos.

(4) Pliometría

Multi-saltos (flexo-extensión de tobillo) ${ }^{1}$, saltos con rodillas al pecho ${ }^{1}$, squat jumps ${ }^{1}$, saltar 3 conos (frontal y lateral $)^{1}$, doble salto unipodal y caída controlada ( 5 reps/pierna), salto horizontal unipodal ( 5 reps/pierna), saltos máximos $(200 \mathrm{ft})$, saltos diagonales profundos $(100 \mathrm{ft})$.

\begin{tabular}{|c|c|}
\hline Ejercicios semana 4 & Duración \\
\hline \multicolumn{2}{|l|}{ (3) Fortalecimiento } \\
\hline $\begin{array}{l}\text { Elevación de talones, plancha frontal y lateral, flexiones, zancadas laterales y diagonales, zancadas con des- } \\
\text { plazamiento, superman para lumbares, superman alternando elevación brazo-pierna contralateral, superman } \\
\text { levantando piernas ( } 90^{\circ} \text { rodillas) y brazos. }\end{array}$ & (3) 30 s/ejercicio \\
\hline \multicolumn{2}{|l|}{ (4) Pliometría } \\
\hline $\begin{array}{l}\left.\text { Multi-saltos (flexo-extensión de tobillo) })^{1} \text {, squat jumps }{ }^{1} \text {, saltar } 3 \text { conos (frontal y lateral }\right)^{1} \text {, saltos laterales profun- } \\
\text { dos (bounding) })^{1} \text {, doble salto unipodal y caída controlada ( } 5 \text { reps/pierna), salto horizontal unipodal ( } 5 \text { reps/pier- } \\
\text { na), saltos diagonales profundos }(100 \mathrm{ft} \text { ). }\end{array}$ & $(4)^{1}$ : Reps en $30 \mathrm{~s}$ \\
\hline
\end{tabular}

Tabla 4. Descripción de la propuesta de ejercicios que presenta actualmente el programa KIPP.

\begin{tabular}{|c|c|}
\hline Ejercicios día de partido & $\begin{array}{l}\text { Repeticiones / } \\
\text { Duración }\end{array}$ \\
\hline 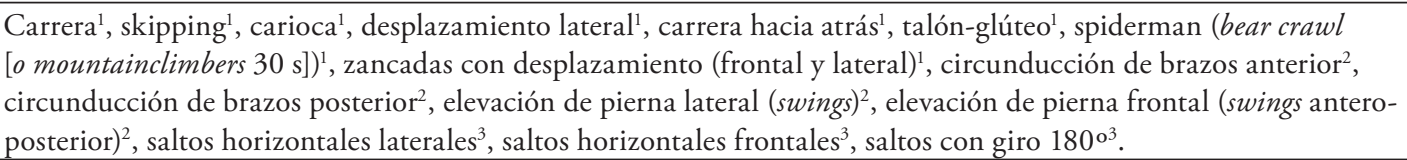 & $\begin{array}{l}{ }^{1}: 100 \mathrm{ft} / \mathrm{ejercicio} \\
{ }^{2}: 10 \mathrm{reps} / \mathrm{ejercicio} \\
{ }^{3}: 30 \text { s/ejercicio }\end{array}$ \\
\hline Ejercicios día de entrenamiento $\mathrm{A}$ & Duración \\
\hline 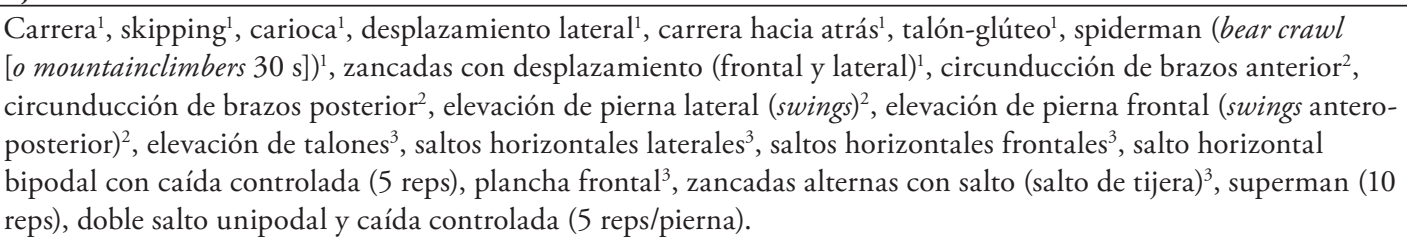 & $\begin{array}{l}{ }^{1}: 100 \mathrm{ft} / \text { ejercicio } \\
{ }^{2}: 10 \text { reps/ejercicio } \\
{ }^{3}: 30 \text { s/ejercicio }\end{array}$ \\
\hline
\end{tabular}




\begin{tabular}{|c|c|}
\hline Ejercicios día de entrenamiento B & Duración \\
\hline 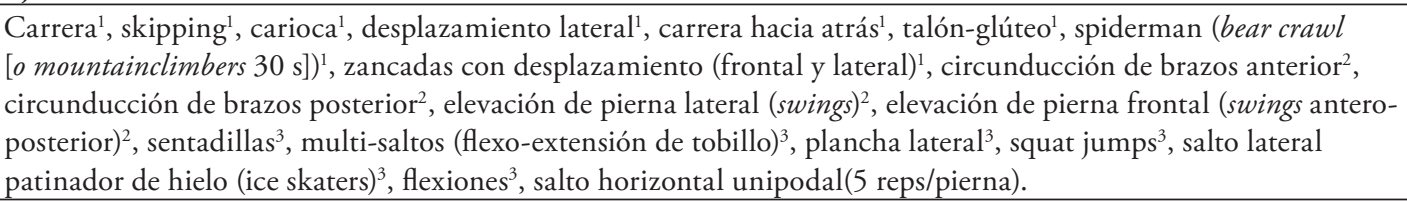 & $\begin{array}{l}{ }^{1}: 100 \mathrm{ft} / \text { ejercicio } \\
{ }^{2}: 10 \mathrm{reps} / \text { ejercicio } \\
{ }^{3}: 30 \text { s/ejercicio }\end{array}$ \\
\hline Ejercicios opcionales para días de entrenamiento (elegir 1 grupo por día) & Duración \\
\hline
\end{tabular}

\section{Grupo 1}

Superman alternando elevación brazo-pierna contralateral (10 reps), zancadas en los tres planos (5 reps/pierna), saltos con rodillas al pecho $(30 \mathrm{~s})$, saltos unipodales profundos (bounding) en el sitio (30 s), carrera aceleracióndeceleración (shuttle run) (50 ft x 10 reps).

Grupo 2

Russian Hamstring (10 reps), saltos diagonales profundos (30 s), saltos máximos (30 s), desplazamientos laterales (50 ft x 10 reps).

$\mathrm{ft}$ : pies; reps: repeticiones; s: segundos.

\section{Knäkontroll}

El programa neuromuscular Knäkontroll (Knäkontroll, SISU Idrottsböcker $($, Sweden, 2005) es un programa de calentamiento estructurado diseñado para disminuir la ratio de lesiones agudas de rodilla en jóvenes deportistas. Se trata de un programa de prevención dirigido a deportes de equipo y desarrollado por un grupo de expertos y experimentados fisioterapeutas pertenecientes a la prestigiosa organización médica de la Asociación Sueca de Fútbol, en colaboración con la Federación de Balonmano, la Federación de Baloncesto y la Federación de Floorball del mismo país (Hägglund, Waldén, \& Atroshi, 2009). Este programa combina 6 ejercicios centrados especialmente en contenidos de fuerza y en el control de la correcta alineación de la rodilla: (1) sentadilla unipodal, (2) puente lumbar dinámico, (3) sentadilla bipodal, (4) plancha frontal, (5) zancadas, y (6) técnica de salto y caída (Tabla 5). Además, cada ejercicio presenta una variante para realizar en parejas que puede ser utilizada de manera intermitente con el objetivo de favorecer la adherencia y variabilidad del programa a través del componente lúdico. Previo a la aplicación de estos ejercicios, los autores recomiendan realizar 5 minutos de carrera continua a baja intensidad. Por tanto, la duración aproximada de la completa implementación del Knäkontroll es de 20 minutos.

Los datos aportados en relación al efecto de esta medida preventiva sobre la incidencia lesional en el deporte parecen positivos; Waldén, Atroshi, Magnusson, Wagner, \& Hägglund (2012) mostraron una reducción del 64\% de la ratio de lesión de LCA en mujeres adolescentes futbolistas. No obstante, se hace necesario investigar los efectos de este programa sobre otras cohortes poblacionales.

Tabla 5. Descripción de ejercicios y duración del programa Knäkontroll.

\begin{tabular}{|c|c|}
\hline Ejercicio & $\begin{array}{l}\text { Repeticiones/ } \\
\text { Duración }\end{array}$ \\
\hline \multicolumn{2}{|l|}{ (1) Sentadilla unipodal (niveles de progresión A, B, C y D) } \\
\hline A) Manos en la cadera & $3 \times 8-15$ reps \\
\hline B) Sosteniendo un balón por encima de la cabeza con brazos estirados & $3 \times 8-15$ reps \\
\hline C) Manos en la cadera; imaginar un reloj y marcar sobre el suelo las 12, 2, 4 y 6 en punto con el pie no ejecutor & $3 \times 5$ reps \\
\hline $\begin{array}{l}\text { D) Sosteniendo un balón con las manos, bajar hasta tocar el suelo y subir en diagonal hasta levantar balón por } \\
\text { encima de la cabeza con brazos estirados hacia el lado contralateral }\end{array}$ & $3 \times 8-15$ reps \\
\hline Pareja) Compañero presiona lateralmente con el balón la pierna libre del ejecutante de la tarea & Pareja) $3 \times 5-10$ reps \\
\hline \multicolumn{2}{|l|}{ (2) Puente lumbar dinámico } \\
\hline A) Bipodal con manos cruzadas en el pecho & $3 \times 8-15$ reps \\
\hline B) Unipodal con manos agarrando pierna libre flexionada a 90 (cadera y rodilla) & $3 \times 8-15$ reps \\
\hline $\begin{array}{l}\text { C) Unipodal pie ejecutor apoyado sobre un balón, pierna libre flexionada a } 90^{\circ} \text { (cadera y rodilla) y manos apoya- } \\
\text { das en el suelo }\end{array}$ & $3 \times 8-15$ reps \\
\hline D) Unipodal, alternando pierna de apoyo manteniendo brazos en el suelo con codos a $90^{\circ}$ & $3 \times 8-15$ reps \\
\hline $\begin{array}{l}\text { Pareja) Compañero agarra el talón del pie mientras que el ejecutante de la tarea utiliza este apoyo para levantar la } \\
\text { pelvis del suelo }\end{array}$ & Pareja) $3 \times 8-15$ reps \\
\hline
\end{tabular}




\begin{tabular}{|c|c|}
\hline Ejercicio & $\begin{array}{l}\text { Repeticiones/ } \\
\text { Duración }\end{array}$ \\
\hline \multicolumn{2}{|l|}{ (3) Sentadilla bipodal } \\
\hline A) Sosteniendo balón con brazos estirados en frente del cuerpo & $3 \times 8-15$ reps \\
\hline B) Manos en la cadera & $3 \times 8-15$ reps \\
\hline C) Sosteniendo un balón por encima de la cabeza con brazos estirados & $3 \times 8-15$ reps \\
\hline $\begin{array}{l}\text { D) Igual que en el nivel C pero terminando el movimiento en posición inicial apoyando únicamente el tercio } \\
\text { distal del pie (elevando talones) }\end{array}$ & $3 \times 8-15$ reps \\
\hline $\begin{array}{l}\text { Pareja) Compańero situado a } 1 \text { metro de distancia y en dirección opuesta al ejecutante; sostener un balón entre } \\
\text { los dos utilizando una mano cada uno y llevando la otra mano apoyada en la cadera. Presionar el balón cuando se } \\
\text { realiza la flexión para la sentadilla }\end{array}$ & Pareja) $3 \times 8-15$ reps \\
\hline \multicolumn{2}{|l|}{ (4) Plancha frontal } \\
\hline A) Apoyando antebrazos y rodillas & $15-30 s$ \\
\hline B) Apoyando antebrazos y pies & $15-30 s$ \\
\hline C) Apoyando antebrazos y moviendo lateralmente el apoyo de los pies de manera alterna hasta volver a posición inicial & $15-30 s$ \\
\hline D) Plancha lateral dinámica & 5-10 reps \\
\hline Pareja) Carretilla & Pareja) $15-30 \mathrm{~s}$ \\
\hline \multicolumn{2}{|l|}{ (5) Zancadas } \\
\hline A) Manos en la cadera & $3 \times 8-15$ reps \\
\hline $\begin{array}{l}\text { B) Sosteniendo un balón con brazos estirados en frente del cuerpo, realizar zancada con rotación del tronco } \\
\text { llevando balón hacia el lado de la pierna ejecutora }\end{array}$ & $3 \times 8-15$ reps \\
\hline C) Sosteniendo un balón por encima de la cabeza con brazos estirados & $3 \times 8-15$ reps \\
\hline D) Sosteniendo un balón con brazos estirados en frente del cuerpo, realizar zancadas laterales & $3 \times 8-15$ reps \\
\hline Pareja) Lanzar balón al compañero situado a 5-10 metros cuando se realiza la zancada & Pareja) $3 \times 8-15$ reps \\
\hline \multicolumn{2}{|l|}{ (6) Salto y caída } \\
\hline A) Saltos unipodales antero-posterior con manos en la cadera & $3 \times 8-15$ reps \\
\hline B) Saltos unipodales laterales alternando pierna ejecutora en cada repetición; manos en la espalda & $3 \times 8-15$ reps \\
\hline C) Dar pequeños pasos en el sitio y realizar salto horizontal unipodal aterrizando con pierna contralateral & $3 \times 5$ reps \\
\hline D) Igual que el nivel C pero realizando cambio de dirección de $90^{\circ}$ antes de ejecutar el salto; alternar lados & $3 \times 5$ reps \\
\hline $\begin{array}{l}\text { Pareja) Compañero situado a } 5 \text { metros lanza el balón para que el ejecutante realice salto bipodal, remate de cabeza } \\
\text { y caiga sobre sus dos piernas }\end{array}$ & Pareja) $3 \times 8-15$ reps \\
\hline
\end{tabular}

PEP

En un esfuerzo por diseñar un programa efectivo que ayudara a prevenir la lesión del ligamento cruzado anterior de la rodilla, el grupo de investigación de la Fundación de Medicina Deportiva de Santa Mónica desarrolló el denominado "Programa de Prevención de Lesiones y Mejora de Rendimiento" (Prevent injury and Enhance Performance Program [PEP]). Este programa tiene por objetivo combatir activamente determinados déficits que puedan presentar jóvenes jugadoras de fútbol (14-18 años) y que impliquen un incremento del riesgo de lesión en la articulación de la rodilla, en general, y en el ligamento cruzado anterior, en particular. Para ello, el programa cuenta con 5 apartados que presentan, a su vez, un total de 19 ejercicios cuyos contenidos principales son la carrera, la flexibilidad, la fuerza, la pliometría y la agilidad (Tabla 6). La duración total de la aplicación del programa es de 15-20 minutos (Mandelbaum et al., 2005).

Además, esta propuesta de programa preventivo presentada por Mandelbaum et al. (2005) podría verse completada con los 5 ejercicios adicionales incluidos por Lim et al. (2009) y englobados dentro de un bloque final denominado "ejercicios alternativos de vuelta a la calma”. Así, a los 19 ejercicios descritos en la Tabla 6, Lim et al. (2009) agregaron: (1) puente lumbar unipodal alternando pierna de apoyo, (2) encorvamiento abdominal, (3) estiramiento lumbar mediante rodillas al pecho, (4) estiramiento del piriforme y (5) estiramiento de aductores. Estos ejercicios podrían situarse, por tanto, como elementos finales dentro del programa preventivo si este se realiza en sesiones aisladas al entrenamiento habitual del deportista, o como vuelta a la calma post-entrenamiento si el programa se desarrolla (tal y como proponen sus autores) como calentamiento previo a la práctica deportiva regular.

El cumplimiento del objetivo principal del PEP ha sido estudiado por Mandelbaum et al. (2005) en mujeres jugadoras de fútbol, mostrando reducciones significativas del número de lesiones de ligamento cruzado anterior de la rodilla en aquellas deportistas que sustituyeron su programa de calentamiento habitual por este programa preventivo. Por tanto, el PEP podría ser una interesante medida a adoptar en aquellos deportistas que presenten evidentes disfunciones o alteraciones motoras y que impliquen un mayor riesgo de lesión de LCA. 
Tabla 6. Descripción de ejercicios y duración del programa PEP.

\begin{tabular}{|c|c|}
\hline Ejercicio & Duración \\
\hline (1) Calentamiento & \\
\hline Carrera en línea recta, carrera lateral, carrera hacia atrás (30 s por ejercicio). & $1.5 \mathrm{~min}$ \\
\hline (2) Estiramientos & \\
\hline Estiramiento de gemelos, cuádriceps, isquiosurales, aductores, flexores de cadera (30 s por pierna y por ejercicio). & $5 \mathrm{~min}$ \\
\hline $\begin{array}{l}\text { (3) Fortalecimiento } \\
\text { Zancadas }(2 \times 20 \mathrm{yd}) \text {, Russian/Nordic Hamstring (30 reps), equilibrio dinámico unipodal (30 reps con cada pierna). }\end{array}$ & $\begin{array}{l}\text { Aproximada- } \\
\text { mente } 3 \text { min }\end{array}$ \\
\hline $\begin{array}{l}\text { (4) Pliometría } \\
\text { Saltos laterales bipodales, saltos antero-posteriores bipodales, saltos antero-posteriores unipodales, saltos verticales, } \\
\text { zancadas alternas con salto ( } 20 \text { reps por ejercicio). }\end{array}$ & $\begin{array}{l}\text { Aproximada- } \\
\text { mente } 2.5 \mathrm{~min}\end{array}$ \\
\hline (5) Agilidad & \\
\hline $\begin{array}{l}\text { Carrera aceleración-deceleración (shuttle run }[40 \text { yd]), carrera diagonal ( } 40 \text { yd), carrera realizando saltos altos monopo- } \\
\text { dales (bounding [ } 45-50 \mathrm{yd}] \text { ) }\end{array}$ & $\begin{array}{l}\text { Aproximada- } \\
\text { mente } 3 \text { min }\end{array}$ \\
\hline
\end{tabular}

min: minutos; s: segundos; reps: repeticiones; yd: yardas.

\section{Eficacia de los programas preventivos descritos}

La publicación de nuevas propuestas para la prevención de lesiones ha ido acompañada, normalmente, del análisis de la eficacia de las mismas. La Tabla 7 resume los resultados aportados por los principales ensayos controlados aleatorios (randomized controlled trials [RTC]) en relación a la eficacia de estas medidas para la prevención de lesiones en jóvenes deportistas.

\section{Principales ventajas e inconvenientes de los programas de prevención analizados}

Todos los programas presentados en esta revisión bibliográfica muestran resultados positivos en términos de reducción de la incidencia lesional en el deporte. Por tanto, todos podrían ser utilizados por los distintos profesionales en su práctica habitual como medida preventiva. No obstante, y para facilitar a los profesionales la elección del programa más adecuado de acuerdo a su contexto de actuación, a continuación se van a analizar las posibles ventajas e inconvenientes que pueden mostrar estos cinco programas en función de algunas de sus principales características.

De las distintas medidas preventivas presentadas, el FIFA 11+ ha sido una de las más estudiadas. Sus efectos positivos sobre la reducción de la incidencia lesional en jóvenes deportistas han quedado ampliamente demostrados incluso en deportes distintos a su población objetivo. Otra de las ventajas de este programa es la subdivisión de los ejercicios del segundo bloque (parte principal) por niveles de progresión, permitiendo la adaptación de la carga y dificultad de la propuesta a las características individuales de los deportistas. La presencia de estos niveles de progresión en las medidas propuestas se hace de vital importancia, pues la aplicación de volúmenes e intensidades inadecuados podría incrementar el riesgo de lesión por sobreuso en nuestros deportistas (Myer et al., 2011). Por el contrario, una posible desventaja de este programa es la ausencia de ejercicios de estiramiento ignorando así el trabajo de flexibilidad como componente protector frente al riesgo de lesión (Dill, Begalle, Frank, Zinder, \& Padua, 2014). 
Tabla 7. Características de los principales estudios que analizan la eficacia de los cinco programas de prevención de lesiones incluidos en esta revisión.

\begin{tabular}{|c|c|c|c|c|c|c|c|}
\hline Programa & Referencia & País & Participantes & Frecuencia & Duración & Grupos & Resultados más significativos \\
\hline \multirow{4}{*}{ FIFA 11+ } & $\begin{array}{l}\text { Longo et al. } \\
\text { (2012) }\end{array}$ & Italia & $\begin{array}{c}121 \text { hombres } \\
\text { jugadores } \\
\text { de balon- } \\
\text { cesto }(M \\
\text { intervención } \\
=13.5 \text { años; } \\
M \text { control = } \\
15.2 \text { años) }\end{array}$ & $\begin{array}{c} \\
\text { 3-4 veces } \\
\text { por semana }\end{array}$ & 9 meses & $\begin{array}{l}\text { Control: calentamien- } \\
\text { to habitual } \\
\text { Intervención: FIFA } \\
11+\end{array}$ & $\begin{array}{l}\text { FIFA } 11+\text { mostró reducción sig- } \\
\text { nificativa de la incidencia lesional } \\
\text { global }(0.95 \text { vs } 2.16 \text { les } / 1000 \mathrm{~h}) \text {, de } \\
\text { la incidencia en EEII ( } 0.68 \text { vs } 1.4 \\
\text { les/1000h), y de la gravedad de las } \\
\text { lesiones ( } 0 \text { vs } 0.51 \text { les/1000h). }\end{array}$ \\
\hline & $\begin{array}{l}\text { Owoeye et } \\
\text { al. }(2014)\end{array}$ & Nigeria & $\begin{array}{l}414 \text { hombres } \\
\text { jugadores de } \\
\text { fútbol (14-19 } \\
\text { años) }\end{array}$ & $\begin{array}{l}2 \text { veces por } \\
\text { semana }\end{array}$ & 6 meses & $\begin{array}{l}\text { Control: calentamien- } \\
\text { to habitual } \\
\text { Intervención: FIFA } \\
11+\end{array}$ & $\begin{array}{l}\text { FIFA } 11+\text { mostró reducción signifi- } \\
\text { cativa de la incidencia lesional global } \\
\text { ( } 0.8 \text { vs } 1.5 \text { les } / 1000 \mathrm{~h}) \text { y de la inciden- } \\
\text { cia en EEII }(0.6 \text { vs } 1.2 \text { les } / 1000 \mathrm{~h}) \text {. }\end{array}$ \\
\hline & $\begin{array}{l}\text { Soligard et } \\
\text { al. }(2008)\end{array}$ & Noruega & $\begin{array}{l}1892 \text { mujeres } \\
\text { jugadoras de } \\
\text { fútbol (13-17 } \\
\text { años) }\end{array}$ & $\begin{array}{l}2 \text { veces por } \\
\text { semana }\end{array}$ & $\begin{array}{l}1 \text { tempo- } \\
\text { rada }\end{array}$ & $\begin{array}{l}\text { Control: calentamien- } \\
\text { to habitual } \\
\text { Intervención: FIFA } \\
11+\end{array}$ & $\begin{array}{l}\text { FIFA } 11+\text { mostró reducción sig- } \\
\text { nificativa de la incidencia lesional } \\
\text { global }(3.23 \text { vs } 4.73 \text { les } / 1000 \mathrm{~h}) \text {, de } \\
\text { las lesiones por sobreuso }(0.50 \text { vs } 1.14 \\
\text { les } / 1000 \mathrm{~h}), \text { y de la gravedad de las } \\
\text { lesiones }(0.16 \text { vs } 0.53 \text { les } / 1000 \mathrm{~h}) \text {. }\end{array}$ \\
\hline & $\begin{array}{l}\text { Steffen et al. } \\
\quad(2013)\end{array}$ & Canadá & $\begin{array}{l}226 \text { mujeres } \\
\text { jugadoras de } \\
\text { fútbol (13- } \\
18 \text { años) }\end{array}$ & $\begin{array}{l}\text { 2-3 veces } \\
\text { por semana }\end{array}$ & 4 meses & $\begin{array}{c}\text { Control: entrenador } \\
\text { desarrolla FIFA 11+ } \\
\text { sin explicación previa } \\
\text { Regular: entrenador } \\
\text { desarrolla FIFA 11+ } \\
\text { tras sesión explicativa. } \\
\text { Supervisado: especia- } \\
\text { lista implementa el } \\
\text { programa } \\
\end{array}$ & $\begin{array}{l}\text { No se encontraron diferencias sig- } \\
\text { nificativas entre grupos. Se observó } \\
\text { reducción significativa de la inciden- } \\
\text { cia lesional entre grupo con alta ad- } \\
\text { herencia al programa ( } 2.7 \text { les/1000h) } \\
\text { y grupo con media adherencia ( } 10.8 \\
\text { les/1000h). }\end{array}$ \\
\hline Harmoknee & $\begin{array}{l}\text { Kiani et al. } \\
\text { (2010) }\end{array}$ & Suecia & $\begin{array}{l}1506 \text { mujeres } \\
\text { jugadoras de } \\
\text { fútbol (13- } \\
19 \text { años) }\end{array}$ & $\begin{array}{l}1-2 \text { veces } \\
\text { por semana }\end{array}$ & $\begin{array}{l}1 \text { tempo- } \\
\text { rada }\end{array}$ & $\begin{array}{l}\text { Control: calentamien- } \\
\text { to habitual } \\
\text { Intervención: Har- } \\
\text { moknee }\end{array}$ & $\begin{array}{l}\text { Harmoknee presentó elevada } \\
\text { reducción de la incidencia lesional } \\
\text { de la rodilla }(0.04 \text { vs } 0.20 \text { les } / 1000 \mathrm{~h}) \\
\text { y, concretamente, de las lesiones sin } \\
\text { contacto en esta articulación }(0.01 \text { vs } \\
0.15 \text { les } / 1000 \mathrm{~h}) \text {. }\end{array}$ \\
\hline KIPP & $\begin{array}{l}\text { LaBella et al. } \\
\text { (2011) }\end{array}$ & EEUU & $\begin{array}{l}1492 \text { mujeres } \\
\text { jugadoras de } \\
\text { fútbol y ba- } \\
\text { loncesto ( } M \\
\text { intervención } \\
\text { y control = } \\
16.2 \text { ańos) }\end{array}$ & $\begin{array}{l}3 \text { veces por } \\
\text { semana }\end{array}$ & 3 meses & $\begin{array}{l}\text { Control: calentamien- } \\
\text { to habitual } \\
\text { Intervención: KIPP }\end{array}$ & $\begin{array}{l}\text { KIPP obtuvo reducción significa- } \\
\text { tiva de la incidencia de lesiones sin } \\
\text { contacto en EEII (especialmen- } \\
\text { te: lesiones agudas [0.71 vs } 1.61 \\
\text { les } / 1000 \mathrm{~h}] \text {, por sobreuso }[0.43 \mathrm{vs} \\
1.22 \text { les } / 1000 \mathrm{~h}] \text {, esguince de tobillo } \\
{[0.25 \mathrm{vs} 0.74 \text { les } / 1000 \mathrm{~h} \text { y y lesiones de }} \\
\text { EEII tratadas quirúrgicamente [0 vs } \\
0.17 \text { les } / 1000 \mathrm{~h}] \text { ) }\end{array}$ \\
\hline $\begin{array}{l}\text { Knäkon- } \\
\text { troll }\end{array}$ & $\begin{array}{l}\text { Waldén et al. } \\
\text { (2012) }\end{array}$ & Suecia & $\begin{array}{l}4564 \text { muje- } \\
\text { res jugadoras } \\
\text { de fútbol } \\
\text { (12-17 ańos) }\end{array}$ & $\begin{array}{l}2 \text { veces por } \\
\text { semana }\end{array}$ & $\begin{array}{l}1 \text { tempo- } \\
\text { rada }\end{array}$ & $\begin{array}{l}\text { Control: calentamien- } \\
\text { to habitual } \\
\text { Intervención: } \\
\text { Knäkontroll } \\
\end{array}$ & $\begin{array}{c}\text { Knäkontroll redujo significativamen- } \\
\text { te la incidencia de lesiones de LCA } \\
(0.05 \text { vs } 0.11 \text { les/1000h). }\end{array}$ \\
\hline PEP & $\begin{array}{l}\text { Mandel- } \\
\text { baum et al. } \\
(2005)\end{array}$ & EEUU & $\begin{array}{c}1041 \text { mujeres } \\
\text { jugadoras de } \\
\text { fútbol (14-18 } \\
\text { años) }\end{array}$ & - & $\begin{array}{l}2 \text { tempora- } \\
\text { das }\end{array}$ & $\begin{array}{l}\text { Control: calentamien- } \\
\text { to habitual } \\
\text { Intervención: PEP }\end{array}$ & $\begin{array}{c}\text { PEP mostró reducción significativa } \\
\text { de la incidencia lesional de LCA en la } \\
\text { temporada } 1 \text { ( } 0.05 \text { vs } 0.47 \text { les } / 1000 \mathrm{~h}) \\
\text { y en la temporada } 2(0.13 \text { vs } 0.51 \\
\text { les } / 1000 \mathrm{~h}) .\end{array}$ \\
\hline
\end{tabular}

M: media; les/1000h: lesiones por cada 1000h de exposición; EEII: extremidades inferiores; LCA: ligamento cruzado anterior. 
El Harmoknee es un programa de prevención que ha demostrado una extraordinaria efectividad en la reducción de la incidencia lesional de la rodilla. Además, a pesar de que los cinco programas analizados presentan un tipo de intervención multicomponente, el Harmoknee es de las propuesta que presentan mayor variabilidad de contenidos en su intervención junto a la de LaBella et al. (2011) y Mandelbaum et al. (2005). Entre sus principales ventajas también destaca la inclusión de un bloque de estiramientos dinámicos. En este sentido, tan solo la propuesta de Mandelbaum et al. (2005) reserva, al igual que esta de Kiani et al. (2010), uno de los bloques de su programa para el trabajo de flexibilidad. Sin embargo, el Harmoknee es el único programa que utiliza técnicas de estiramiento dinámicas, siguiendo así las recomendaciones realizadas por estudios previos que demuestran el superior efecto de estas técnicas sobre el rendimiento motor en relación a las técnicas de estiramiento estático (Ayala \& Sainz de Baranda, 2010; Fletcher \& Jones, 2004; Thompsen, Kackley, Palumbo, \& Faigenbaum, 2007). En cuanto a las principales desventajas destaca la ausencia de niveles de progresión. El amplio rango de edad que engloba este programa requiere de una estratificación por niveles de progresión de los ejercicios, volúmenes e intensidades propuestos. De lo contrario, participantes con notables diferencias en su desarrollo y rendimiento motor recibirán idénticas cargas de entrenamiento, influyendo muy posiblemente en la magnitud de los efectos obtenidos tras su implementación.

El KIPP es un programa que ha reportado efectos beneficiosos sobre la prevención de lesiones de extremidades inferiores en general, aunque su objetivo principal es la reducción de la ratio de incidencia de la rodilla. Entre sus ventajas se encuentra la amplia variabilidad de ejercicios que propone, favoreciendo así la progresión del deportista a lo largo de las distintas semanas de implementación. Esta gran cantidad de ejercicios presenta, al mismo tiempo, uno de los principales inconvenientes del programa; hasta un total de 41 ejercicios se pueden necesitar para el desarrollo íntegro de la medida en la tercera semana de aplicación, una cifra excesivamente elevada y poco práctica para su uso en el día a día.

Otro de los programas descritos en este artículo ha sido el Knäkontroll, SISU Idrottsböcker@. Las ventajas de este programa son similares a las del FIFA 11+: la presencia de varios niveles de progresión $(\mathrm{n}=4)$ y de un reducido número de ejercicios $(n=6)$ hacen de este un programa de gran utilidad a efectos prácticos. Además, la propuesta de ejercicios alternativos para desarrollar en pareja favorece el aspecto lúdico del mismo. Por tanto, se trata de una buena opción para desarrollar en la práctica deportiva habitual. Como principal inconveniente se podría destacar la ausencia de ejercicios para el trabajo de flexibilidad.

Finalmente, el PEP ha sido otra de las alternativas publicadas en los últimos años. Al igual que el resto de programas, su efectividad ha sido estudiada previamente mostrando resultados notablemente positivos. La simplicidad de su estructura y de los ejercicios propuestos hace de este programa una buena alternativa a los programas de calentamiento tradicionales. Sin embargo, como ya se ha comentado anteriormente, aunque incluye un bloque de contenidos para el trabajo de flexibilidad, la utilización de estiramientos estáticos (30 s/pierna) como técnica principal podría no ser positiva para el posterior rendimiento del deportista, constituyendo así uno de los inconvenientes más destacables de este programa.

\section{Conclusiones}

Todos los programas presentados en esta revisión bibliográfica muestran resultados positivos en términos de reducción de la incidencia lesional en el deporte. Por tanto, todos podrían ser utilizados por los distintos profesionales en su práctica habitual como medida preventiva. No obstante, tras el análisis pormenorizado de la evidencia científica, se concluye que los programas FIFA 11+ y el Knäkontroll son los más recomendados. Ambos han demostrado ser programas muy efectivos, presentan varios niveles de progresión (entre 3 y 4 niveles) que favorecen la adaptación del programa al contexto de aplicación y, en base al número (entre 6 y 15 ejercicios), tipo de ejercicios (no requieren de material adicional) y duración (10$20 \mathrm{~min}$ ), facilitan su implementación de manera regular en el deporte.

\section{Referencias bibliográficas}

1. Ayala, F., \& Sainz de Baranda, P. (2010). Efecto agudo del estiramiento sobre el sprint en jugadores de fútbol de división de honor juvenil. Revista Internacional de Ciencias Del Deporte, 6(18), 1-12. http://doi. org/10.5232/ricyde2010.01801

2. Beachy, G., \& Rauh, M. (2014). Middle school injuries: A 20-year (1988-2008) multisport evaluation. Journal of Athletic Training, 49(4), 493-506. http://doi.org/10.4085/1062-6050-49.2.19

3. Daneshjoo, A., Mokhtar, A. H., Rahnama, N., \& Yusof, A. (2012). The Effects of Comprehensive Warm-Up Programs on Proprioception, Static and Dynamic Balance on Male Soccer Players. PLoS ONE, 7(12), 1-10. http://doi.org/10.1371/journal.pone.0051568
4. Daneshjoo, A., Mokhtar, A. H., Rahnama, N., \& Yusof, A. (2013). The effects of injury prevention warm-up programmes on knee strength in male soccer players. Biology of Sport, 30(4), 281-288. http://doi. org/10.5604/20831862.1077554

5. Daneshjoo, A., Rahnama, N., Mokhtar, A. H., \& Yusof, A. (2013). Effectiveness of injury prevention programs on developing quadriceps and hamstrings strength of young male professional soccer players. Journal of Human kKnetics, 39, 115-25. http://doi.org/10.2478/hukin-2013-0074

6. Dill, K. E., Begalle, R. L., Frank, B. S., Zinder, S. M., \& Padua, D. A. (2014). Altered knee and ankle kinematics during squatting in 
those with limited weight-bearing-lunge ankle-dorsiflexion range of motion. Journal of Athletic Training, 49(6), 723-732. http://doi. org/10.4085/1062-6050-49.3.29

7. Fletcher, I. M., \& Jones, B. (2004). The Effect of Different Warm-Up Stretch Protocols on 20 Meter Sprint Performance in Trained Rugby Union Players. Journal of Strength and Conditioning Research, 18(4), 885-888. http://doi.org/10.1519/14493.1

8. Hägglund, M., Waldén, M., \& Atroshi, I. (2009). Preventing knee injuries in adolescent female football players - design of a cluster randomized controlled trial. BMC Musculoskeletal Disorders, 10, 75. http://doi. org/10.1186/1471-2474-10-75

9. Hootman, J. M., Dick, R., \& Agel, J. (2007). Epidemiology of collegiate injuries for 15 sports: Summary and recommendations for injury prevention initiatives. Journal of Athletic Training, 42(2), 311-319.

10. Janssen, I., \& Leblanc, A. (2010). Systematic Review of the Health Benefits of Physical Activity and Fitness in School-Aged Children and Youth. International Journal of Behavioral Nutrition and Physical Activity, 7(40), 1-16. http://doi.org/10.1201/b18227-14

11. Kiani, A., Hellquist, E., Ahlqvist, K., Gedeborg, R., Michaëlsson, K., \& Byberg, L. (2010). Prevention of soccer-related knee injuries in teenaged girls. Archives of Internal Medicine, 170(1), 43-49. http://doi. org/10.1001/archinternmed.2009.289

12. LaBella, C. R., Huxford, M. R., Grissom, J., Kim, K.-Y., Peng, J., \& Christoffel, K. K. (2011). Effect of Neuromuscular Warm-up on Injuries in Female Soccer and Basketball Athletes in Urban Public High Schools: Cluster Randomized Controlled Trial. Archives of Pediatrics and Adolescent Medicine, 165(11), 1033-1040. http://doi.org/10.1001/ archpediatrics. 2011.168

13. Lim, B.-O., Lee, Y. S., Kim, J. G., An, K. O., Yoo, J., \& Kwon, Y. H. (2009). Effects of sports injury prevention training on the biomechanical risk factors of anterior cruciate ligament injury in high school female basketball players. The American Journal of Sports Medicine, 37(9), 1728-1734. http://doi.org/10.1177/0363546509334220

14. Longo, U. G., Lamberti, A., Maffulli, N., \& Denaro, V. (2011). Tissue engineered biological augmentation for tendon healing: A systematic review. British Medical Bulletin, 98(1), 31-59. http://doi.org/10.1093/ $\mathrm{bmb} / \mathrm{ldq} 030$

15. Longo, U. G., Loppini, M., Berton, A., Marinozzi, A., Maffulli, N., \& Denaro, V. (2012). The FIFA 11+ program is effective in preventing injuries in elite male basketball players: a cluster randomized controlled trial. The American Journal of Sports Medicine, 40(5), 996-1005. http:// doi.org/10.1177/0363546512438761
16. Loprinzi, P. D., Cardinal, B. J., Loprinzi, K. L., \& Lee, H. (2012) Benefits and environmental determinants of physical activity in children and adolescents. Obesity Facts, 5(4), 597-610. http://doi. org/10.1159/000342684

17. Maffulli, N., Longo, U. G., Gougoulias, N., Loppini, M., \& Denaro, V. (2010). Long-term health outcomes of youth sports injuries. British Journal of Sports Medicine, 44(1), 21-25. http://doi.org/10.1136/ bjsm.2009.069526

18. Mandelbaum, B. R., Silvers, H. J., Watanabe, D. S., Knarr, J. F., Thomas, S. D., Griffin, L. Y., ... Garrett, Wi. J. (2005). Effectiveness of a Neuromuscular and Proprioceptive Training Program in Preventing the Incidence of Anterior Cruciate Ligament Injuries in Female Athletes : 2-year follow up. The American Journal of Sports Medicine, 33(7), 1-8. http://doi.org/10.1177/0363546504272261

19. Myer, G. D., Faigenbaum, A. D., Ford, K. R., Best, T. M., Bergeron, M. F., \& Hewett, T. E. (2011). When to initiate integrative neuromuscular training to reduce sports-related injuries and enhance health in youth? Current Sports Medicine Reports, 10(3), 155-166. http://doi. org/10.1249/JSR.0b013e31821b1442

20. Owoeye, O. B. A., Akinbo, S. R. A., Tella, B. A., \& Olawale, O. A (2014). Efficacy of the FIFA 11+Warm-Up Programme in Male Youth Football: A Cluster Randomised Controlled Trial. Journal of Sports Science and Medicine, 13(2), 321-328.

21. Soligard, T., Myklebust, G., Steffen, K., Holme, I., Silvers, H., Bizzini, M., ... Andersen, T. E. (2008). Comprehensive warm-up programme to prevent injuries in young female footballers: cluster randomised controlled trial. BMJ (Clinical Research Ed.), 337, a2469.

22. Steffen, K., Emery, C. A., Romiti, M., Kang, J., Bizzini, M., Dvorak, J., ... Meeuwisse, W. H. (2013). High adherence to a neuromuscular injury prevention programme (FIFA 11+) improves functional balance and reduces injury risk in Canadian youth female football players: a cluster randomised trial. British Journal of Sports Medicine, 47(12), 794-802. http://doi.org/10.1136/bjsports-2012-091886

23. Thompsen, A. G., Kackley, T. E. D., Palumbo, M. A., \& Faigenbaum, A. D. (2007). Acute effects of different warm-up protocols with and without a weighted vest on jumping performance in athletic women. The Journal of Strength \& Conditioning Research, 21(1), 52-56.

24. Waldén, M., Atroshi, I., Magnusson, H., Wagner, P., \& Hägglund, M. (2012). Prevention of acute knee injuries in adolescent female football players: cluster randomised controlled trial. BMJ (Clinical Research Ed.), 344, 1-11. http://doi.org/10.1136/bmj.e3042 